\title{
Diagnosis within a Diagnosis: An Unusual Case of Intracranial Hypertension
}

\author{
Emma Foster $^{1, *}$, Mastura Monif ${ }^{1,2}$, Kylie Mason $^{3}$, Anneke van der Walt ${ }^{1,4}$ \\ ${ }^{1}$ Department of Neurology, The Royal Melbourne Hospital, Parkville, Australia \\ ${ }^{2}$ Department of Physiology, University of Melbourne, Parkville, Australia \\ ${ }^{3}$ Department of Haematology, The Royal Melbourne Hospital, Parkville, Australia \\ ${ }^{4}$ Melbourne Brain Centre at The Royal Melbourne Hospital, Department of Medicine, University of Melbourne, Parkville, Australia \\ *Corresponding author
}

\begin{abstract}
A 56 year old man presented with several months of new onset headaches and blurred vision. Lumbar puncture revealed elevated intracranial pressure, but multiple cerebral imaging studies failed to identify a secondary cause. Eventually a formal venogram revealed a transverse venous sinus thrombosis, and an occult malignancy screen led to a diagnosis of non-Langerhans histiocytosis, or Erdheim-Chester Disease. This case highlights two important learning points: 1) secondary causes for raised intracranial pressure should be vigorously sought in atypical cases of idiopathic intracranial hypertension; 2) ECD is a rare but important cause of ophthalmologic and neurologic presentations, and should be a differential for orbital infiltration, intra- and extracranial disease.
\end{abstract}

Keywords: Papilloedema, Erdheim-Chester Disease, histiocytosis, optic nerve fenestration, elevated intracranial pressure, proto-oncogene protein B-raf

Cite This Article: Emma Foster, Mastura Monif, Kylie Mason, and Anneke van der Walt, "Diagnosis within a Diagnosis: An Unusual Case of Intracranial Hypertension.” Neuro-Ophthalmology \& Visual Neuroscience, vol. 2, no. 1 (2017): 1-3. doi: 10.12691/novn-2-1-1.

A 56 year old male presented to his family doctor with frequent headaches and blurred vision. His past medical history consisted of the following: (1) systemic hypertension, (2) surgically treated left retinal detachment 28 years prior with residual visual acuity of 6/24 in that eye, and (3) hyperopia managed with reading glasses. His body mass index was 22.5. He was on no regular medication, and denied use of tobacco, alcohol, or recreational drugs. There was no past history of venous sinus thrombosis, clotting disorder, or previous brain surgery. He was referred to our hospital's Neuro-ophthalmology department after a routine optometry review noted bilateral disc oedema. Relevant examination findings included Frisen grade 1-2 papilledema (Figure 1) and reduced visual acuity of 6/9 in the right eye and 6/24-1 in the left eye.

A lumbar puncture revealed an opening pressure of $30 \mathrm{cmH}_{2} \mathrm{O}$. Cerebrospinal fluid (CSF) analysis was unremarkable, with normal protein count. Neither cerebral computer tomography (CT) or magnetic resonance imaging (MRI) venograms revealed evidence of a venous sinus thrombosis or other secondary cause for his raised intracranial pressure (ICP). Stenosis of the left transverse sinus was observed but was not thought to be contributing to his clinical presentation. MRI of the orbits was unremarkable. Routine blood tests were unremarkable.
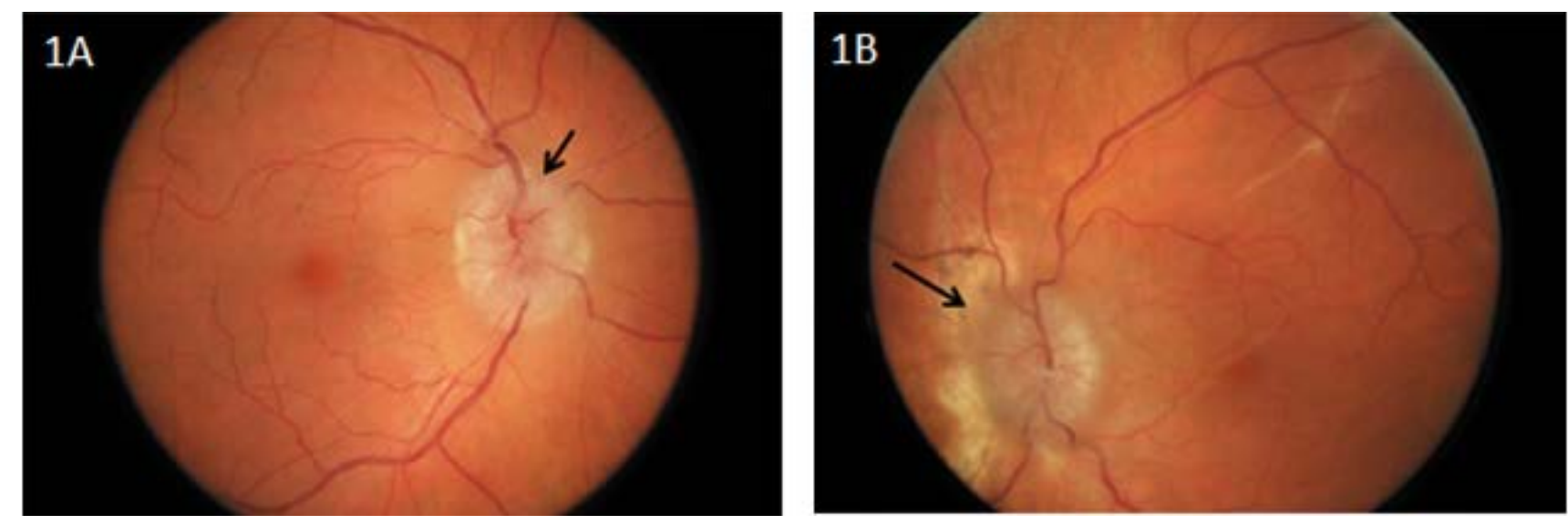

Figure 1. Fundoscopic images of right $(1 \mathrm{~A})$ and left (1B) eyes. Arrows point to blurred optic disc margins and oedema, more significant on the left 


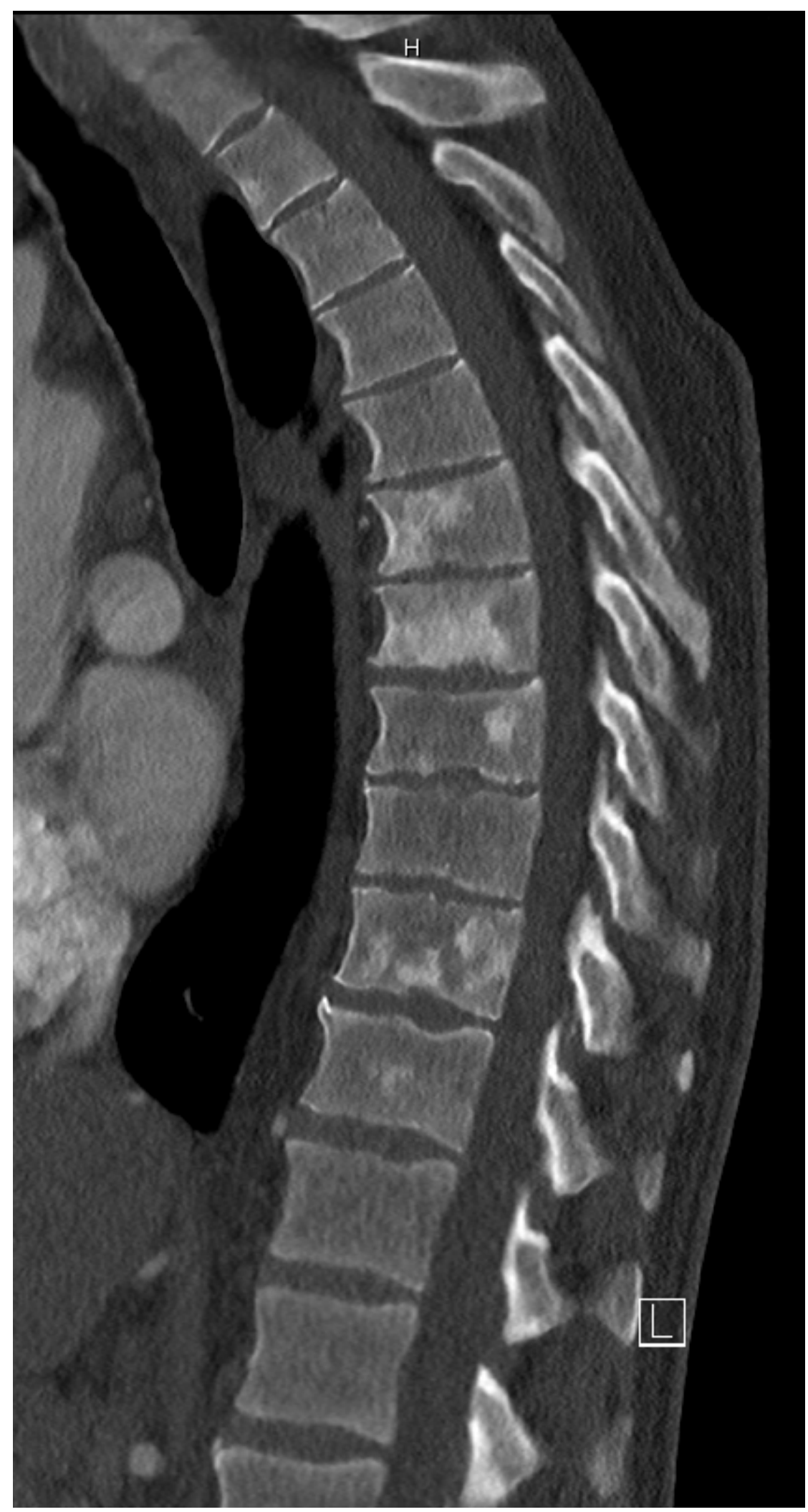

Figure 2. Multiple sclerotic lesions involving the thoracic vertebral bodies

Despite the atypical presentation, he was diagnosed with possible idiopathic intracranial hypertension (IIH) and commenced on acetazolamide 250mg twice daily. Monthly reviews, and, two further lumbar punctures, revealed stable visual acuity and consistently raised ICP $\left(30 \mathrm{~cm} \mathrm{H}_{2} \mathrm{O}\right)$. The CSF biochemistry and cell count was repeatedly normal. Acetazolamide was up titrated to 500mg twice daily. Four months later he noted further subacute reduction in visual acuity in the left eye. Examination revealed visual acuity (left eye) 6/60, new left eye relative afferent pupillary defect, and new left eye red-green colour blindness, consistent with severe left optic neuropathy. Fundoscopy revealed grade 3-4 papilledema bilaterally. He underwent urgent left optic nerve fenestration, improving visual acuity (left eye) to $6 / 24$. The diagnosis was revisited and a formal venogram was performed that revealed a left transverse venous sinus 
thrombosis. He was anticoagulated with warfarin. A screen for prothrombotic conditions, including an occult malignancy, was conducted. CT thorax, abdomen, and pelvis revealed multiple vertebral sclerotic lesions (Figure 2), retroperitoneal fibrosis, and aortic wall thickening.

This was consistent with histiocytotic disease. Bone Marrow Aspirate and Trephine were consistent with inflammatory change and demonstrated no cytogenetic abnormality and were negative for BRAF. Biopsy of a sclerotic bone lesion in the pelvis revealed histiocytes that were CD68 positive, CD1a negative, and BRAF V600E mutation positive. These findings were consistent with non-Langerhans histiocytosis, or Erdheim-Chester disease (ECD). Given the absence of systemic symptoms, he was commenced on interferon alfa 2a, and continues to attend regular Neuro-ophthalmology and Haematology reviews.

ECD is a rare clonal neoplastic disorder first described in 1930 by scientists Jakob Erdheim and William Chester [1]. Although the exact frequency is unknown, epidemiological studies reveal a male preponderance, and a mean age at diagnosis of 55 years [2]. The affected cells are thought to express proinflammatory substances, chemokines and cytokines, driving local activation and recruitment of histiocytes. This leads to a self-propagating, pathological, inflammatory response, and subsequent organ dysfunction. ECD is commonly associated with an oncogenic BRAFV600E mutation, found in 38-68\% of cases [3]. B-Raf proteins have a role in regulating signalling pathways, which affects cell division, differentiation, and secretion. BRAF abnormalities are driver mutations in several malignancies. Diagnosis is confirmed by specific histopathologic findings, in the appropriate clinical and radiological context [3]. Distinctive histological features include foamy, lipid-laden histiocytes with surrounding fibrosis. Immunohistochemical staining is generally positive for CD68 (a marker of macrophages, monocytes, and histiocytes), CD163 (a marker of histiocytes and macrophages), and Factor XIIIa (marker of fibrohistiocytic precursor cells), and helps differentiate from diseases such as Langerhans cell histiocytosis. A multicentre observational cohort study revealed that skeletal involvement is almost universal, affecting 96\% of patients [2], with cardiac and pulmonary involvement occurring in approximately half of patients [3]. Central nervous system (CNS) involvement is also common, and occurs in $51.5 \%$ of patients [2]. Parenchymal CNS involvement has serious implications, associated with both clinically significant disability and increased risk of death [2]. ECD-related neurological symptoms range from those caused by compression of local structures through to generalised deterioration of cognition and gait [3]. Orbital involvement, with infiltration of one or both orbits, occurs in approximately $25 \%$ of cases [2]. These patients present with exophthalmos, retro-orbital pain, oculomotor palsies, or blindness [3]. A retrospective review of pathologically confirmed ECD cases revealed orbital disease in $32 \%$ of patients who underwent neuroimaging [4]. Other common radiological features include osteosclerosis in long bones such as femur and tibia, seen almost universally, perinephric stranding or 'hairy kidney' appearance, and periaortic infiltration [3]. There are few prospective studies [2] and no randomised control trials regarding treatment of ECD. Consensus guidelines recommend starting therapy over expectant management, with the largest amount of evidence available for interferon-a-2a and pegylated interferon [3]. BRAFV600E-inhibitors are currently under investigation for those with oncogenic mutations, and may significantly impact prognosis and survival which currently stands at $68 \%$ at 5-year follow up [2].

This is a fascinating case with several important learning points. Firstly, the patient's sex, age and physical phenotype made him an atypical for classic idiopathic intracranial hypertension and investigations for secondary causes were actively pursued. Multiple MR venograms were negative, and digital subtraction angiography (DSA) was needed before the cause for the raised ICP was demonstrated. CT venogram has a reported sensitivity of $95 \%$ compared to DSA as the gold standard; other studies have found that CT venogram is at least equivalent to $M R$ venogram in the diagnosis of venous sinus thrombosis [5]. A secondary cause for raised ICP should be vigorously sought if there is a clinical suspicion for an underlying pathology. Secondly, ECD is a rare but important cause of ophthalmologic and neurologic presentations. Neuro-ophthalmologists, Neurologists, and Ophthalmologists should be aware of the variety of ways in which it may manifest, and it should be a differential diagnosis for orbital infiltration, as well as extra- and intracranial disease.

\section{Conflict of Interest Statement}

No relevant disclosures for any authors.

\section{Fund}

No funding was received for this work.

\section{References}

[1] Chester, W., "Lipidgranulomatose", Virchows Arch Pathol Anat, 279, 561-602, 1930

[2] Arnaud, L., Hervier, B., Neel, A., Hamidou, M. A., Kahn, J-E., Wechsler, B. Perez-Pastor, G., Blomberg, B., Fuzibet, J-G., Dubourguet, F., Marinho, A., Magnette, C., Noel, V., Pavic, M., Casper, J., Beucher, A-B., Costedoat-Chalumeau, N., Aaron, L., Salvatierra, J., Graux, C., Cacoub, P., Delcey, V., Dechant, Cl., Bindi, P., Herbaut, C., Graziani, G., Amoura, Z., and Haroche, J., "CNS involvement and treatment with interferon-a are independent prognostic factors in Erdheim-Chester disease: a multicentre survival analysis of 53 patients", Blood, 117(10), 2778-2782, March 2011.

[3] Diamond, E.L., Dagna, L., Hyman, D., Cavalli, G., Janku, F., Estrada-Veras, J., Ferrarini, M. Abdel-Wahab, O., Heaney, M.L., Scheel, P.J., Feeley, N.K., Ferrero, E., McClain, K.L., Vaglio, A., Colby, T., Arnaud, L., Haroche, J., "Consensus guidelines for the diagnosis and clinical management of Erdheim-Chester disease", Blood, 124(4), 483-492, July 2014.

[4] Parks, N., "Neurologic and cranial manifestations of ErdheimChester disease", American Academy of Neurology Annual Meeting (Poster), P2.390, April 2017.

[5] Alvis-Miranda, H. R., Castellar-Leones, S. M., Alcala-Cerra, G., Moscote-Salazar, L. R., "Cerebral sinus venous thrombosis", $J$ Neurosci Rural Pract, 4(4), 427-438, Oct-Dec 2013. 